\title{
Refining Exoplanet Ephemerides and Transit Observing Strategies
}

\author{
Stephen R. Kane, ${ }^{1}$ Suvrath Mahadevan, ${ }^{2}$ Kaspar von Braun,,${ }^{1}$ Gregory Laughlin, ${ }^{3}$ and David R. Ciardi ${ }^{1}$ \\ Received 2009 August 27; accepted 2009 September 27; published 2009 November 16
}

\begin{abstract}
Transiting planet discoveries have yielded a plethora of information regarding the internal structure and atmospheres of extrasolar planets. These discoveries have been restricted to the low-periastron distance regime due to the bias inherent in the geometric transit probability. Monitoring known radial velocity planets at predicted transit times is a proven method of detecting transits, and presents an avenue through which to explore the massradius relationship of exoplanets in new regions of period/periastron space. Here we describe transit window calculations for known radial velocity planets, techniques for refining their transit ephemerides, target selection criteria, and observational methods for obtaining maximum coverage of transit windows. These methods are currently being implemented by the Transit Ephemeris Refinement and Monitoring Survey (TERMS).
\end{abstract}

\section{INTRODUCTION}

Planet formation theories thus far extract much of their information from the known transiting exoplanets, which are largely in the short-period regime. This is because transit surveys that have provided the bulk of the transiting planet discoveries, such as SuperWASP (Pollacco et al. 2006) and HATNet (Bakos et al. 2002), are biased toward this region. The two planets that contribute to the sample of intermediate to longperiod transiting exoplanets are: HD 17156b (Barbieri et al. 2007) and HD 80606b (Laughlin et al. 2009; Moutou et al. 2009), the latter of which exhibits both a primary transit and secondary eclipse. In both of these cases, the detection was largely due to the inflated transit/eclipse probability caused by their extreme orbital eccentricities. Both of these were observed to transit through predictions based upon their radial velocity data.

Planetary orbits may be considered in three basic categories: short-period $(<10$ days) planets, intermediate to long-period high-eccentricity $(>0.1)$ planets, and intermediate to longperiod low-eccentricity planets. The first type of planet is the focus of current studies, and we are beginning to gain insight into the second type (HD 80606b, for example). Exploration into the structure of those planets occupying the second and third orbit types will require probing parameter-space beyond that currently encompassed by the known transiting exoplanets. The science objectives of such an exploration include understanding how planetary properties, such as average planet density, vary with periastron distance, as well as providing the first observational data for exoplanet models with low incident

\footnotetext{
'NASA Exoplanet Science Institute, Caltech, Pasadena, CA 91125; skane@ ipac.caltech.edu.

${ }^{2}$ Department of Astronomy and Astrophysics, Pennsylvania State University, University Park, PA 16802

${ }^{3}$ UCO/Lick Observatory, University of California, Santa Cruz, CA 95064.
}

stellar radiation. Recent observations of HD 80606b by Gillon (2009) and Pont et al. (2009) suggest a spin-orbit misalignment caused by a Kozai mechanism; a suggestion which could be investigated in terms of period and eccentricity dependencies if more long-period transiting planet were known.

Figure 1 shows the distribution of periastron distances for the known exoplanets using data from Jean Schneider's Extrasolar Planets Encyclopaedia ${ }^{4}$. Also shown as a shaded histogram is the same distribution for the known transiting exoplanets. Clearly the periastron distribution of the known transiting planets does not accurately represent the distribution of the entire sample of known exoplanets. Thus far, our picture of exoplanets is based on the planets with superheated atmospheres through either short-period orbits or intermediate-period eccentric orbits. Figure 1 shows how our view of planetary properties is highly biased toward planets of short periastron distance, thus demonstrating the need for detecting transits of exoplanets at larger distances in order to study atmospheres with greatly reduced flux from the parent star.

Long-period transits will give us valuable insight into the structure of exoplanets that are more similar to those in our own solar system. Additionally, host stars of long-period planets discovered using the radial velocity technique tend to be very bright, and potentially one of these could be the brightest star with a transiting Jupiter-mass planet. The brightness of such host stars can faciliate atmospheric studies via transmission spectra (Burrows et al. 2006; Redfield et al. 2008; Snellen et al. 2008) and thermal phase variations (Knutson et al. 2007). The long period typically also translates to a longer transit duration, allowing significantly more signal to be collected in attempts to probe the atmospheric composition of these objects with highresolution spectrographs. Since most bright late F, G, K-type

\footnotetext{
${ }^{4}$ At http://exoplanet.eu/.
} 


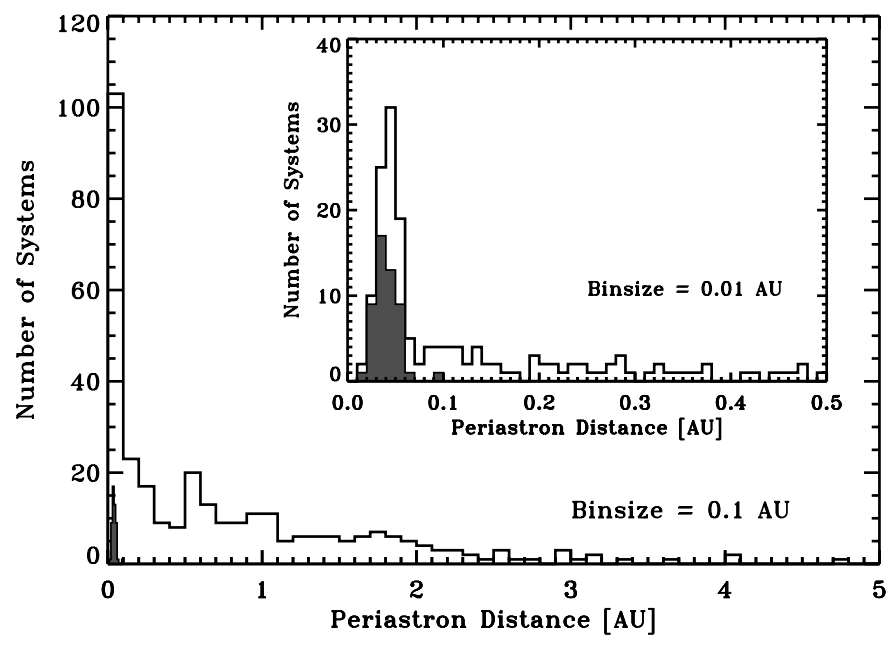

FIG. 1.-Histogram of periastron distances of known exoplanets. The shaded region corresponds to known transiting planets, the population of which is comprised of planets in short-period orbits and intermediate-period eccentric orbits.

stars have already been searched for short-period Jupiter-mass planets, the long-period hosts are the obvious sample to now search since their relative brightness and longer transit times are both very helpful in attempting to probe the exoplanetary atmosphere.

It is clear that the discovery of long-period transiting planets is very important. They are, however, difficult to detect in ground-based transit surveys due to the presence of correlated (red) noise (Pont et al. 2006) and effects of the observational window function (von Braun et al. 2009). Though combining data from various ongoing transit surveys to search for transiting long-period planets will improve detectability (Fleming et al. 2008), it is easier to discover transits among the large number of long-period exoplanets already known from radial velocity surveys. Based upon Monte Carlo simulations of the transit probabilities, it is expected that several of the known longperiod planets should transit their parent stars due to the enhanced probabilities produced by eccentric orbits (Barnes 2007; Kane \& von Braun 2008). There have been suggestions regarding the strategy for photometric follow-up of these radial velocity planets at predicted times of primary transit (Kane 2007) and secondary eclipse (Kane \& von Braun 2009), and the instruments which could be used for such surveys (LópezMorales 2006). Here we describe a methodology through which to search for transits of known planets. In $\S 2$ we calculate transit windows for a large selection of the known exoplanets and show the impact of additional radial velocity measurements on refining the transit ephemerides. Section 3 describes the techniques through which optimal target selection and observations of the transit window can be achieved. The methods described here have been successfully tested and implemented by the Transit Ephemeris Refinement and Monitoring Survey (TERMS).

\section{TRANSIT WINDOWS}

The transit window as described here is defined as a specific time period during which a complete transit (including ingress and egress) could occur for a specified planet. The limiting factor for successfully observing a known exoplanet host star during the predicted transit window is often the precision of the transit ephemeris. The quality of the transit ephemeris is primarily determined by (a) the uncertainties associated with the fitted orbital parameters, and (b) the time elapsed since the most recent radial velocity data was acquired. Acquiring new highprecision radial velocity data can easily mitigate both effects. With a prompt photometric observing strategy after the orbital parameters have been revised, one can maximize the chances of being able to obtain complete coverage of the transit observing window and thus either confirm or rule out the transiting nature of the planet.

\subsection{Ephemeris Calculation}

The orbital parameters measured from fitting the radial velocity data of a planet are sufficient for calculating a transit ephemeris. The predicted time of midtransit can be calculated by using Kepler's equations. Firstly, the eccentric anomaly is calculated from the following

$$
E=2 \tan ^{-1}\left(\sqrt{\frac{1-e}{1+e}} \tan \frac{f}{2}\right)
$$

where $e$ is the orbital eccentricity and $f$ is the true anomaly. As described in Kane (2007), the time of transit midpoint will occur when $\omega+f=\pi / 2$, where $\omega$ is the argument of periastron. Substituting this for the true anomaly in equation (1) thus yields the eccentric anomaly at the point of predicted transit.

The mean anomaly, $M$, which defines the time since last periapsis in units of radians, is then computed by

$$
M=E-e \sin E
$$

which can be converted to regular time units using

$$
t_{M}=\frac{P M}{2 \pi}
$$

where $P$ is the orbital period. The predicted midpoint of primary transit can then be calculated using

$$
t_{\text {mid }}=t_{p}+\frac{P M}{2 \pi}+n P
$$

where $t_{p}$ is the time of periastron passage and the term of $n \times P$ incorporates the number of complete orbits which have transpired since $t_{p}$.

The uncertainties in the orbital parameters (assuming they are symmetrical) can be propagated through these equations 
to determine the uncertainty in the predicted transit midpoint, $\delta t_{\text {mid }}$, and the size of the transit window, $t_{\text {win }}$. Note that these uncertainties will be equivalent to $1 \sigma$ if the orbital parameter uncertainties are also $1 \sigma$. The size of a transit window is mostly dependent upon the uncertainty in the period and the time elapsed since last observations were acquired. Thus, the beginning and end of a transit window are calculated by subtracting and adding (respectively) the uncertainties in $t_{p}$ and $P$ with respect to the transit midpoint, taking into account the number of orbits since periastron passage and the transit duration. The beginning of a particular transit window can be approximated by

$$
t_{\text {begin }}=\left(t_{p}-\delta t_{p}\right)+(P-\delta P) \frac{M}{2 \pi}+n(P-\delta P)-\frac{t_{d}}{2}
$$

where $\delta t_{p}$ and $\delta P$ are the uncertainties in $t_{p}$ and $P$ respectively, and $t_{d}$ is the transit duration. Conversely, the end of the transit window is approximated by

$$
t_{\mathrm{end}}=\left(t_{p}+\delta t_{p}\right)+(P+\delta P) \frac{M}{2 \pi}+n(P+\delta P)+\frac{t_{d}}{2} .
$$

Hence, the size of a given transit window is defined by subtracting equation (5) from equation (6), resulting in

$$
t_{\text {win }}=2\left(\delta t_{p}+\delta P \frac{M}{2 \pi}+n \delta P\right)+t_{d}
$$

which reduces to simply the transit duration as the uncertainties in $P$ and $t_{p}$ approach zero, as expected. It is clear from equation (7) that $\delta P$ has the potential to rapidly dominate the size of the transit window if the number of orbits since discovery becomes sufficiently large. Equation 7 may be re-expressed as

$$
\delta P=\frac{\pi\left(t_{\mathrm{win}}-t_{d}-2 \delta t_{p}\right)}{M+2 \pi n},
$$

which can be used to determine the period uncertainty needed in order to achieve a certain transit window for a fixed $\delta t_{p}$ and $t_{d}$ (see $\S 2.3$ for examples).

These equations serve as first-order approximations which ignore the uncertainties in the orbital parameters of eccentricity and argument of periastron and instead focus on the timedomain parameters of period and time of periastron passage. However, the equations also serve to overestimate the size of the transit window (the conservative approach) by assuming that the orbital inclination is edge-on compared with the line of sight. The consequence of this is that the maximum transit duration is allowed for.

\subsection{Orbital Parameter Dependencies}

As previously mentioned, the quality of the transit windows depends upon the uncertainties in the orbital parameters. The transit windows also grow with time, prompting follow-up of the transit window as soon as possible after discovery. In this section, we describe these effects for 245 known exoplanets for which the equations in $\S 2.1$ have been used to calculate their transit ephemerides.

Figure 2 shows the size of the first transit window (the first transit to occur after $t_{p}$ ) and the uncertainty in the transit midpoint for the 245 exoplanets in the sample. Also shown are the predicted transit durations and geometric transit probabilities for these exoplanets. These calculations are based on the errors in the orbital parameters derived from currently available radial velocity data.

The necessity of the logarithmic scale in these plots demonstrates the large range in the size of the transit window. The transit windows of the short-period planets tend to be significantly smaller since, at the time of discovery, many orbits have been monitored to provide a robust estimate of the orbital period. This is particularly obvious in the plot of the transit midpoint uncertainies which shows that periods less than $\sim 10$ days have far superior constraints on the calculated ephemerides. In contrast, the longer period exoplanets often only have one orbit completely monitored and it is possible, though uncommon, for the resulting transit window to become comparable to the orbital period of the planet. The ideal targets to monitor in an observing campaign tend to occupy the lower-right corner of the plot of transit window as a function of transit probability. These planets have the highest likelihood of yielding successful detections, though this population is dominated by short-period planets.

Figure 3 shows the net increase in the size of the transit windows for this sample of exoplanets by comparing the first transit windows after discovery with the first transit window occurring after a JD of 2,454,979.5 (CE 2009 May 28 00:00 UT). The open circles shown in the lower-right area of the figure are those long-period planets for which an additional transit window beyond $t_{p}$ has not yet occurred and so the size of the transit window remains unchanged. Note that the distribution of points in this plot now resembles the distribution shown in the transit midpoint uncertainty plot of Figure 2, since the transit duration estimate is unaffected by the passage of time. Therefore, the transit window size increase for the short-period planets is much slower over time than for the long-period planets. This indicates that, even though many more orbits of the short-period planets have occurred, the transit midpoint uncertainty remains dominated by the uncertainty in the period. The size of the transit window for the long-period planets can be brought into a managable regime for photometric follow-up with relatively small usage of large telescope time. Without such an effort, it clear from these plots that it will be impossible to ascertain whether or not many of the long-period planets transit their host stars.

\subsection{Improvements from Additional Data}

As described in $\S 2.2$, a considerable number of high transit probability targets are not feasible (depending upon telescope access) to observe because the uncertainty in the predicted 

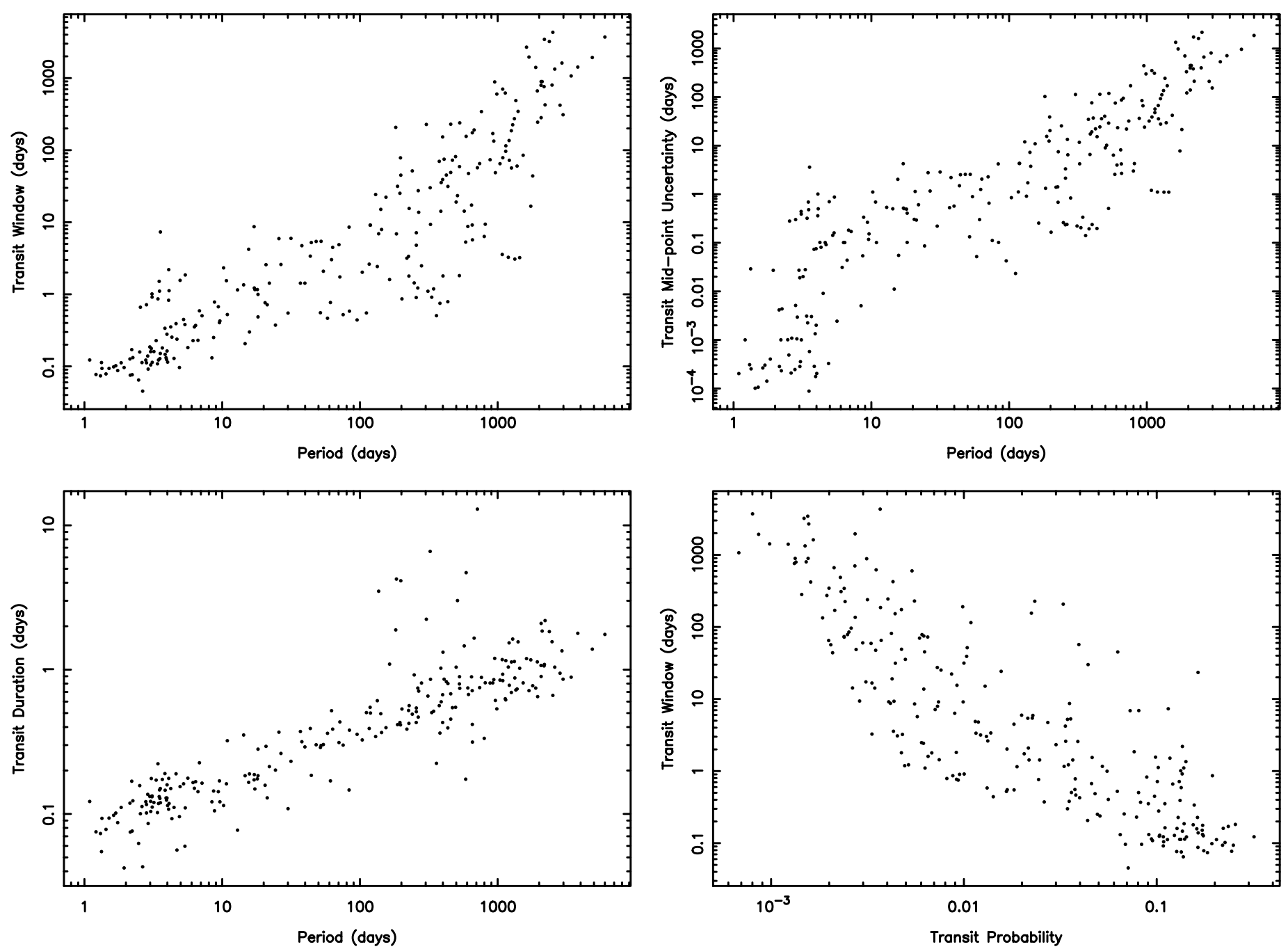

FIG. 2.-Ephemeris calculations for the sample of 245 exoplanets for the first predicted transit after $t_{p}$ (see $\S 2.2$ ). These show the dependence of transit window (top left), transit midpoint uncertainty (top right), and predicted transit duration (bottom left) on period, as well as the relation between the transit window and the transit probability (bottom right).

transit midpoint is too high to justify the observing time required. This can lead to transit windows of months and even years in duration. The acquisition of just a handful of new radial velocity measurements at carefully optimized times can reduce the size of a transit window by an order of magnitude. Here we describe, by way of two examples, how obtaining further radial velocity measurements for known exoplanets can improve the transit ephemerides. These examples were chosen based upon their very different periods, relatively high transit probabilities, availability of radial velocity data, and different transit windows and discovery dates. In each of the examples, we have simulated four additional measurements by using the best-fit orbital parameters to determine the radial velocity at later epochs and adopting the mean of the discovery data precision for the simulated measurement uncertainties. The simulated measurements were then passed through a gaussian filter, which produced scat- ter consistent with the uncertainties, then appended to the discovery data.

\subsubsection{HD 190228}

The planet orbiting the star HD 190228 was discovered by Perrier et al. (2003) as part of a group of new planets announced by the ELODIE team. The planet is in a $~ 1146$ day orbit around a $G$ subgiant star with an eccentricity of $\sim 0.5$. The eccentric nature of the orbit resulted in no radial velocity data being acquired by the discovery team when the planet was close to periapsis, since the planet spends a very small portion of its orbit near that location. Calculations for the first predicted transit to occur after JD 2,454,979.5 (see Fig. 3) yield a transit midpoint uncertainty of 88.9 days and a transit window of 178.9 days. The geometric transit probability of this planet is $\sim 1 \%$ which 


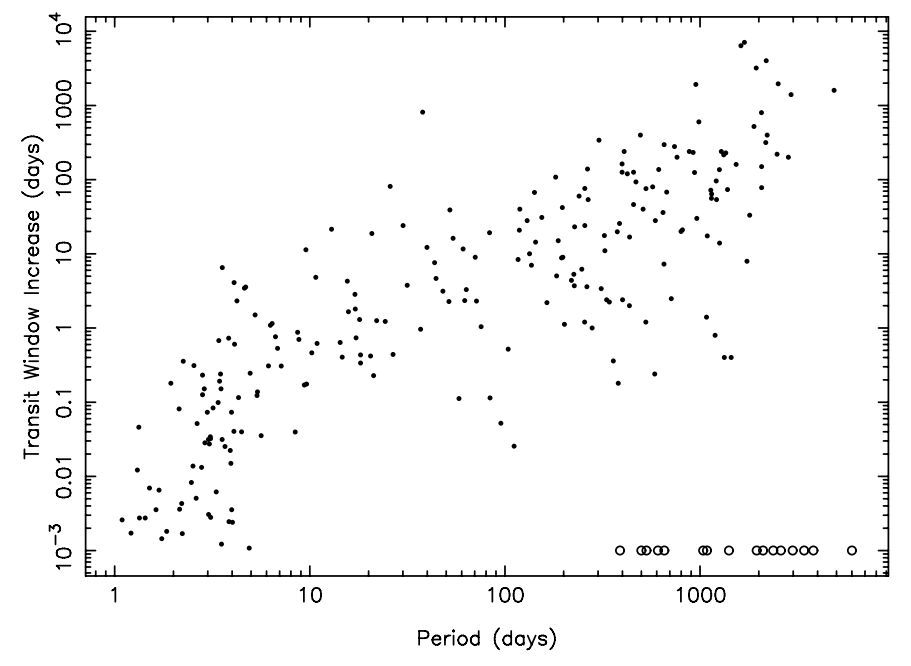

FIG. 3.-Increase in the size of the transit window from the predicted time of the first window after $t_{p}$ to the predicted window after a JD of 2,454,979.5. The open circles shown in the bottom right are those long-period planets for which an additional transit window beyond $t_{p}$ has not occurred as of this JD.

is relatively high for a planet of this orbital period. However, the large transit window makes this an unfeasible target to observe, particularly from the ground where a substantial fraction of the total transit window will remain uncovered (assuming only one ground-based telescope at a particular longitude is being used).

In Figure 4 we show the discovery data of Perrier et al. (2003) along with four additional simulated measurements. The simulated data are each separated from each other by 50 days. Note that the simulated measurements have been acquired while the planet is speeding past periapsis. The peri-

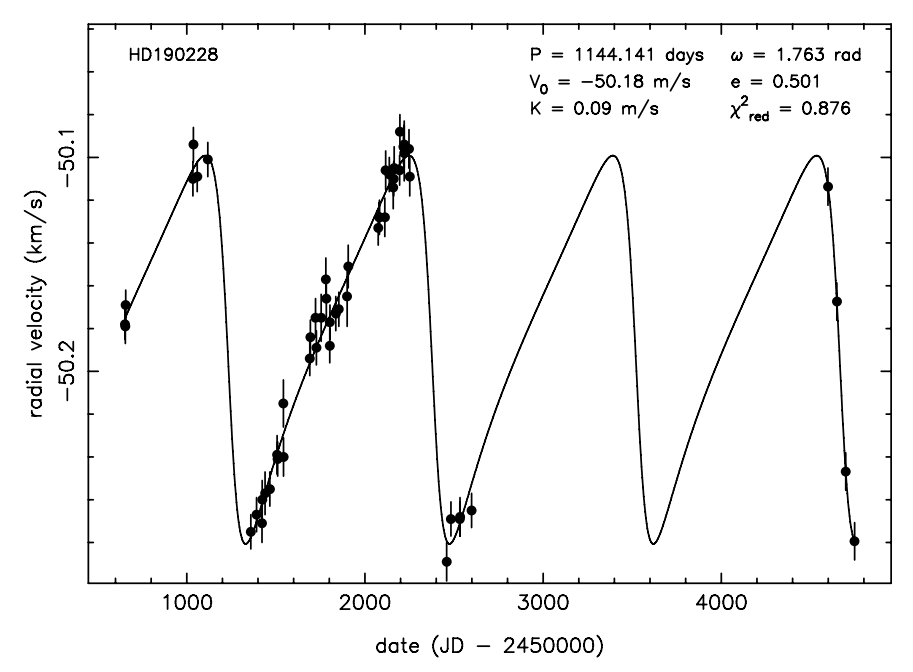

FIG. 4.-Best-fit solution (solid line) to the original radial velocity data of HD 190228 obtained by Perrier et al. (2003) and four subsequent simulated measurements. astron passage of an orbit, particularly for a highly eccentric orbit, is where the planet is moving the fastest and so occupies a relatively small fraction of the total phase space. Thus, the greatest constraints during the shortest period of time can be made by sampling this part of the orbit. These effects have been discussed at length in the context of the effects of eccentric orbits on period analysis (Cumming 2004), cadence optimization for radial velocity surveys (Kane et al. 2008), and adaptive scheduling algorithms (Ford 2008).

The orbital parameters of HD 190228 were recomputed from the combination of discovery and simulated data using the method described by Kane et al. (2007) and Kane et al. (2009). The original and revised orbital parameters are shown in Table 1, based upon the fits to the original and revised data sets respectively. The revised orbital parameters have only slight improvements in their uncertainties with the exception of the period and the time of periastron passage, which are the two most important parameters for calculating the transit window. This improvement from only four additional measurements decreases the uncertainty in the transit midpoint and the size of the transit window (for the first transit to occur after JD $2,454,979.5$ ) by a factor of $\sim 6$. Though the transit window is still quite large (31.0 days), it is now far more accessible, and of course it can be improved further by increasing the phase coverage and time baseline of the radial velocity data. The main benefit to constraining the transit window will come through improving the baseline (measurements during the same phase at subsequent orbits) rather than additional phase coverage, since phase coverage mainly aids toward constraining the shape (eccentricity and periastron argument) of the radial velocity variation.

It should be noted that this analysis does not take into account the more typical situation where the additional measurements are acquired with a different telescope and/or template spectrum than the discovery data. In this case, a floating offset

TABLE 1

FIT PARAMETERS FOR HD 190228B

\begin{tabular}{|c|c|c|}
\hline Parameter & Original Fit & Revised Fit \\
\hline$P$ (days) $\ldots .$. & $1146 \pm 16$ & $1144.14 \pm 2.09$ \\
\hline$V_{0}\left(\mathrm{~km} \mathrm{~s}^{-1}\right) \ldots \ldots$ & $-50.182 \pm 0.004$ & $-50.181 \pm 0.003$ \\
\hline$K\left(\mathrm{~km} \mathrm{~s}^{-1}\right) \quad \ldots \ldots \ldots$ & $91 \pm 5$ & $90.75 \pm 4.36$ \\
\hline$\omega\left(^{\circ}\right) \ldots \ldots \ldots \ldots \ldots \ldots$ & $100.7 \pm_{3.2}^{2.9}$ & $101.03 \pm 4.14$ \\
\hline e $\ldots \ldots \ldots \ldots \ldots \ldots \ldots$ & $0.499 \pm_{0.024}^{0.047}$ & $0.501 \pm 0.041$ \\
\hline$t_{p}(\mathrm{JD} 2,450,000) \ldots$. & $1236 \pm 25$ & $4672.076 \pm 9.085$ \\
\hline$t_{d}$ (days) $\ldots \ldots \ldots \ldots$ & 1.155 & 1.152 \\
\hline$\delta t_{\text {mid }}$ (days) $\ldots \ldots \ldots$ & 88.9 & 14.9 \\
\hline$t_{\text {win }}($ days $) \quad \ldots \ldots \ldots . .$. & 178.9 & 31.0 \\
\hline
\end{tabular}

NoTE.-The original orbital parameters for HD $190228 \mathrm{~b}$ as measured by Perrier et al. (2003) and the revised orbital parameters with the four addditional measurements, along with original and revised transit duration, transit midpoint uncertainty, and transit window. 
(whereby the radial velocity offset between datasets is included as a free parameter) between data sets will need to be applied during the fitting process, such as that described by Wright \& Howard (2009). This, however, has a negligible effect on the accuracy of the fitted orbital parameters, provided that the additional measurements have suitable phase coverage.

\subsubsection{HD 231701}

A more recent planet discovery is that of HD 231701b by Fischer et al. (2007). This planet has an orbital period of $\sim 141$ days with an eccentricity of $\sim 0.1$. Even so, the slight eccentricity and an argument of periastron near $90^{\circ}$ gives the planet an elevated geometric transit probability of $\sim 1.3 \%$. The host star for this planet is a late-F dwarf. The data acquired at discovery was sufficient to constrain the orbital period to within a couple of days. However, enough time has transpired since discovery such that the first predicted transit after JD $2,454,979.5$ has a midpoint uncertainty of 40.9 days and a total transit window of 82.3 days.

Figure 5 shows the discovery data published by Fischer et al. (2007) along with four additional simulated radial velocity measurements. The simulated data are each separated from each other by 10 days. As was the case for HD 190228, we found that the optimized constraint on the period resulted from spacing the new measurements to cover a large range of radial velocity (amplitude) space rather than phase space. This comes at the expense of refining the shape of the periodic variation which, as described earlier, is determined by $e$ and $\omega$. The results from performing a fit to the combined dataset are shown in Table 2. The significant improvement to both the precision of the period and time of periastron passage parameters results in a subsequent improvement to the uncertainty in transit midpoint and

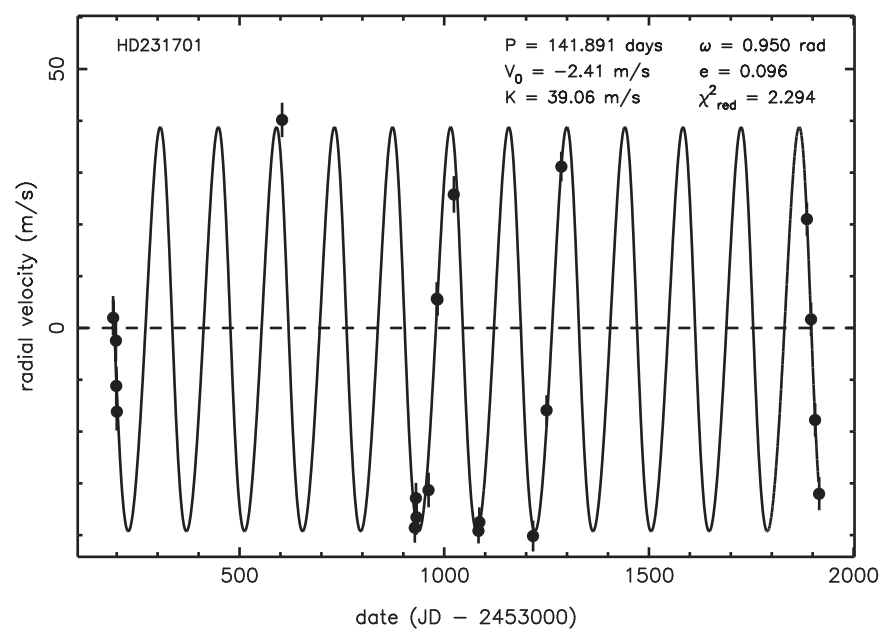

FIG. 5.-Best-fit solution (solid line) to the original radial velocity data of HD 231701 obtained by Fischer et al. (2007) and four subsequent simulated measurements.
TABLE 2

Fit PARAMETERS FOR HD 231701в

\begin{tabular}{|c|c|c|}
\hline Parameter & Original Fit & Revised Fit \\
\hline$P$ (days) . & $141.6 \pm 2.8$ & $141.89 \pm 0.15$ \\
\hline$V_{0}\left(\mathrm{~m} \mathrm{~s}^{-1}\right)$ & & $-2.413 \pm 1.824$ \\
\hline$K\left(\mathrm{~m} \mathrm{~s}^{-1}\right)$ & $39.0 \pm 3.5$ & $39.06 \pm 2.64$ \\
\hline$\omega\left(^{\circ}\right) \ldots .$. & $46 \pm 24$ & $54.40 \pm 3.69$ \\
\hline e & $0.10 \pm 0.08$ & $0.096 \pm 0.069$ \\
\hline$t_{p}(\mathrm{JD} 2,450,000) \ldots \ldots$ & $3180.0 \pm 4.2$ & $4885.141 \pm 1.422$ \\
\hline$t_{d}$ (days) $\ldots \ldots \ldots \ldots \ldots$ & 0.495 & 0.491 \\
\hline$\delta t_{\text {mid }}$ (days) $\ldots \ldots \ldots$ & 40.9 & 1.6 \\
\hline$t_{\text {win }}$ (days) $\quad \ldots \ldots \ldots \ldots$ & 82.3 & 3.7 \\
\hline
\end{tabular}

NOTE.-Original orbital parameters for HD 231701b as measured by Fischer et al. (2007) and the revised orbital parameters with the four additonal measurements, along with original and revised transit duration, transit midpoint uncertainty, and transit window.

transit window size that is impressive-a factor of almost 25 ! This would result in the first transit window beyond JD $2,454,979.5$ being a highly accessible window to obtain good coverage, particularly if longitude coverage could be achieved through appropriate collaborations.

\section{PHOTOMETRIC FOLLOW-UP STRATEGY}

Among the southern hemisphere RV planets, there are few that have been adequately monitored photometrically to confirm or rule out planetary transits. By calculating transit ephemerides for these planets and designing an efficient observing program, it is possible to examine a large subset of these planets with a relatively small amount of observing time on a $1.0 \mathrm{~m}$ class telescope. By applying strict criteria on the predicted transit properties of the targets, we are able to produce a robust selection that yields the most promising targets on which one can place transit constraints. These criteria and general observing strategy considerations are described here.

\subsection{Target Selection}

For each observing run, the experimental design constitutes the selection of targets which meets the necessary criteria (described in this section) for successful observations to be undertaken. The primary challenge is to match transit windows with observability for each target which is not a trivial task. Here we describe the minimum criteria that must be met for each target. The first step is to select all known radial velocity targets for which an estimate of the stellar radius, either from measurement or models, is available for calculating the transit depth and the geometric transit probability. The probability that is most important for the target scheduling is the transit detection probability, which is a combination of the geometric transit probability and the fraction of the transit window during which the target is observable. To first order, this is a straight multiplication but it will 
depend upon the probability distribution of the predicted transit midpoint, as discussed in $\S 3.2$. Based upon the photometric precision of the experimental system, the predicted transit depth can be used to exclude targets whose depth is too low, particularly giant host stars.

The steps thereafter depend upon how the observing time is allocated; a fixed time slot (such as NOAO time), or queuescheduled/service time (such as that used by Kane et al. 2009). For a fixed time slot, the essential steps are:

1. For $0.9-1.0 \mathrm{~m}$ class telescopes, stars brighter than $V=6.0$ often need to be excluded unless the telescope has the options of aperture diaphragms or neutral density filters.

2 . Include only those stars whose airmass is less than $\sim 2$ for at least $3 \mathrm{hr}$ during the night. A transit window of $3 \mathrm{hr}$ is the likely minimum transit window available and so the visibility of the target will still be useful if the transit window happens to largely coincide.

3. For each target that passes the visibility and brightness criteria, the transit ephemeris is checked and transit times are noted for those predicted transits which fall on dates during the run.

4. For each date during the run, the UT times of the transit are checked and transit windows which occur during the day are rejected. Transit windows which do not coincide with the observable hours are also rejected.

5. The schedule for each night is considered in terms of the transit detection probability and the targets are ranked in descending order of the probability. If one seeks to concentrate the investigation on long-period planets, then the planets should be ranked by the observability of the transit windows since the geometric transit probability will dominate the transit detection probability for short-period planets.

Queue-scheduled observations essentially allow an opportunity to target long-period planets whose transit windows occur far less frequently than those usually monitored during preallocated observing runs. For queue-scheduled observations the steps are as follows:

1. Rank the exoplanets from long-period to short-period and keep only those whose transit probability exceeds the geometric transit probability for a circular orbit (see Kane \& von Braun 2008).

2. Examine the transit window for each planet and reject those for which the transit windows are excessively long (for example, greater than $\sim 5$ days).

3. Investigate the visibility of each target from the observing site and reject the targets for which there is a mismatch between the occurrence of the transit window and the time the target is observable. Ideal targets are those for which the transit window occurs during a single night and the target is up all night. For targets which have transit windows spanning multiple nights, the decision will be based upon the value of the target in terms of the transit detection probability.
These steps are almost the reverse of the steps recommended for fixed time-slot observing runs. Since queue-scheduled observations allow for targeted observations of rare transit windows for long-period planets, they are thus those which will yield the highest success for these high-risk/high-return targets.

\subsection{Coverage of Transit Window}

The difficulties in establishing an optimal observing schedule not withstanding, there are further considerations that one needs to take into account when planning observations. One of these is the decision about which parts of the transit window to monitor if the window spans more than one night (and often several nights for long-period planets), especially if one or more of those nights competes with other favorable targets. So far we have assumed that all parts of the transit window are equally significant in the likelihood of a transit being observed or ruled out. This is generally not true but depends upon the probability distribution of the orbital parameter uncertainties. For example, we can suggest an empirical model whereby we assume Gaussian uncertainties for the fit parameters, which in turn assumes Gaussian noise in the radial velocity measurements.

Figure 6 shows a Gaussian probability distribution for the predicted location of the transit midpoint for a planet which has a transit window size $5 \%$ of the orbital period. If one is able to monitor the target for only half of the transit window, then choosing this range to be centered on the predicted transit midpoint (shown as the shaded region in Fig. 6) will account for $38 \%$ of the area under the probability distribution, as opposed to $30 \%$ for the remainder of the time within the transit window.

In reality, the uncertainties associated with the orbital parameters will have a more complex distribution due to systematic

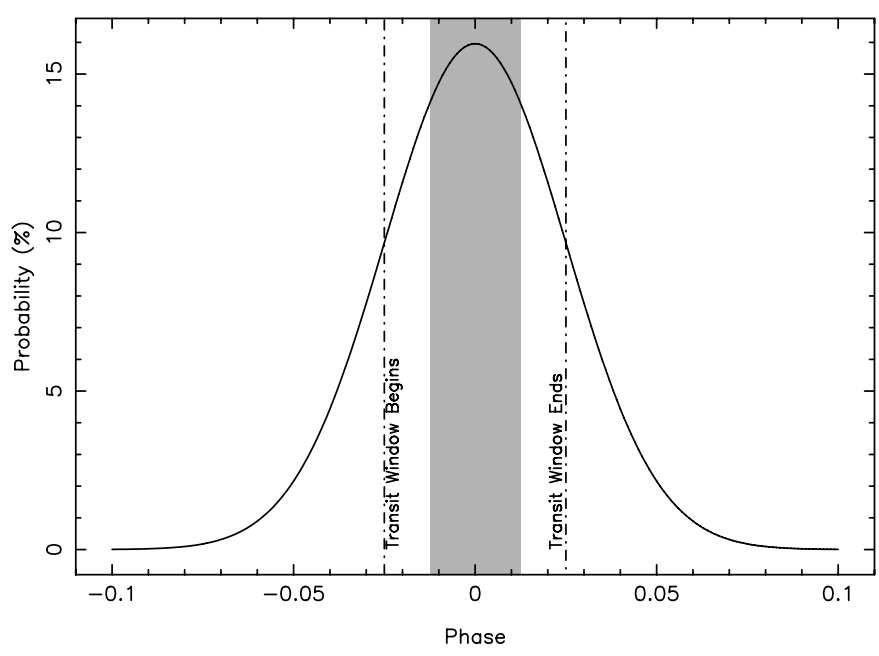

FIG. 6.-Probability distribution for the predicted location of the transit midpoint (as described in $\S 3.2$ ), assuming that the uncertainties in the orbital parameters are Gaussian. 
noise components. Their distributions may be close to that described by Gaussian or Poisson statistics, but can be determined empirically through Monte Carlo simulations which randomize the sequence of the residuals on the radial velocity measurements and redetermining the orbital fit (see Ford 2005 and Kane et al. 2007 for more details). In addition, the probability distribution for a particular parameter is usually nonsymmetric in nature, as seen in a $\chi^{2}$ map of the parameter while the other parameters are kept fixed (for example, see $\chi^{2}$ maps by Kane et al. 2009), but can be approximated as symmetric at the $1 \sigma$ level. Whatever the distribution, the measurements per unit time will be more valuable the closer they are to the predicted midpoint. The caveat to this is when the predicted transit duration is larger than the time for which the target is observable during the night, since it is important to observe either ingress or egress for relative photometry. Thus, if the transit duration is greater than the observing window then the optimal approach is to observe at $t_{\text {mid }} \pm t_{d} / 2$.

The coverage of the transit window can be increased through the use of telescopes adequately separated in longitude. Such networks are already in existence (e.g., the Las Cumbres Observatory Global Telescope/LCOGT network), and collaborations for the follow-up of gamma-ray bursts and microlensing events are quite common. Queue scheduling of observations is particularly useful for the rare transit windows of long-period planets. This kind of observing is available, for instance, to member consortiums who utilize the service time of the Observing with Small and Moderate Aperture Research Telescope System (SMARTS).

For the especially bright targets which will not only saturate typical $1.0 \mathrm{~m}$ class telescopes but whose field-of-view will also be devoid of comparison stars, a solution is to use the Microvariability and Oscillations of Stars (MOST) satellite (Walker et al. 2003; Matthews et al. 2004). The MOST satellite has demonstrated photometric precision of a few parts per million which is sufficient for detecting transit signatures due to planets orbiting bright giant stars. Since MOST is space based, this would also allow complete coverage of the transit window without the need for coordinated ground-based observations using different telescopes (an additional source of red noise). If the transit window can be provided with sufficient accuracy, this would be an excellent use of the MOST satellite's capabilities.

\section{CONCLUSIONS}

Many of the known radial velocity planets have yet to be surveyed for transit signatures. The detection of a transit for the intermediate to long-period planets would add enormously to our knowledge of planetary structure and, in particular, how the structure varies with semimajor axis and periastron distance. The advantages of targeting long-period radial velocity planets are the brightness of the host stars and the prior knowledge of the planetary orbital parameters. However, a major challenge of monitoring the host stars at predicted transit times is that many transit windows have deteriorated over time, such that the telescope time required renders attempts to do so impractical.

We have shown through calculations for 245 of the known exoplanets how the size of the transit window varies with period and geometric transit probability. The large uncertainties associated with the transit midpoint for the long-period planets is dominated by the uncertainties in the period and time of periastron passage estimated from the discovery data. We demonstrated, using the examples of HD 190228 and HD 231701, that a handful of carefully timed additional measurements can vastly improve the size of the transit window and thus bring the monitoring of the window into the reach of ground-based programs.

The difficulties involved in the observing schedule largely result from matching transit windows with the observability of the targets, particularly for long-period planets whose transit windows are widely spaced. We have described a planning strategy which will make optimal use of both preallocated and service telescope time, also noting the advantages of both longitude coverage and space-based observations. It is important to consider for the scheduling that the central part of the transit window can be significantly more valuable than the wings of the window, depending upon the nature of the orbital parameter uncertainties.

The described techniques and science goals are currently being undertaken and investigated by the Transit Ephemeris Refinement and Monitoring Survey (TERMS). Note that the observations from this survey will lead to improved exoplanet orbital parameters and ephemerides even without an eventual transit detection for a particular planet. The results from this survey will provide a complimentary dataset to the fainter magnitude range of the Kepler mission (Borucki et al. 2009), which is expected to discover many transiting planets including those of intermediate to long-period planets.

The authors would like to thank Steven Berukoff for several useful suggestions. This research has made use of the NASA/ IPAC/NExScI Star and Exoplanet Database, which is operated by the Jet Propulsion Laboratory, California Institute of Technology, under contract with the National Aeronautics and Space Administration.

\section{REFERENCES}

Bakos, G. Á., Lázár, J., Papp, I., Sári, P., \& Green, E. M. 2002, PASP, 114, 974

Barbieri, M., et al. 2007, A\&A, 476, L13
Barnes, J. W. 2007, PASP, 119, 986

Borucki, W. J., et al. 2009, Science, 325, 709

Burrows, A., Sudarsky, D., \& Hubeny, I. 2006, ApJ, 650, 1140 
Cumming, A. 2004, MNRAS, 354, 1165

Fischer, D. A., et al. 2007, ApJ, 669, 1336

Fleming, S. W., Kane, S. R., McCullough, P. R., \& Chromey, F. R. 2008, MNRAS, 386, 1503

Ford, E. B. 2005, ApJ, 129, 1706

- 2008, ApJ, 135, 1008

Gillon, M. 2009, MNRAS, preprint (arXiv:0906.4904)

Kane, S. R. 2007, MNRAS, 380, 1488

Kane, S. R., Ford, E. B., \& Ge, J. 2008, IAU Symp., 249, 115

Kane, S. R., Mahadevan, S., Cochran, W. D., Street, R. A., Sivarani, T., Henry, G. W., \& Williamson, M. H. 2009, ApJ, 692, 290

Kane, S. R., Schneider, D. P., \& Ge, J. 2007, MNRAS, 377, 1610

Kane, S. R., \& von Braun, K. 2008, ApJ, 689, 492 2009, PASP, 121, 1096

Knutson, H. A., et al. 2007, Nature, 447, 183

Laughlin, G., Deming, D., Langton, J., Kasen, D., Vogt, S., Butler, P., Rivera, E., \& Meschiari, S. 2009, Nature, 457, 562
López-Morales, M. 2006, PASP, 118, 716

Matthews, J. M., Kusching, R., Guenther, D. B., Walker, G. A. H., Moffat, A. F. J., Rucinski, S. M., Sasselov, D., \& Weiss, W. W. 2004, Nature, 430, 51

Moutou, et al. 2009, A\&A, 498, L5

Perrier, C., Sivan, J.-P., Naef, D., Beuzit, J. L., Mayor, M., Queloz, D., \& Udry, S. 2003, A\&A, 410, 1039

Pollacco, D. L., et al. 2006, PASP, 118, 1407

Pont, F., Zucker, S., \& Queloz, D. 2006, MNRAS, 373, 231

Pont, F., et al. 2009, A\&A, 502, 695

Redfield, S., Endl, M., Cochran, W. D., \& Koesterke, L. 2008, ApJ, 673, L87

Snellen, I. A. G., Albrecht, S., de Mooij, E. J. W., \& Le Poole, R. S. 2008, A\&A, 487, 357

von Braun, K., Kane, S. R., \& Ciardi, D. R. 2009, ApJ, 702, 779

Walker, G. A. H., et al. 2003, PASP, 115, 1023

Wright, J. T., \& Howard, A. W. 2009, ApJS, 182, 205 